\title{
Un roman graphique aux rayons $\mathrm{X}$
}

Johanna Schipper

\section{OpenEdition}

\section{Journals}

Édition électronique

URL : http://journals.openedition.org/genesis/1694

DOI : 10.4000/genesis. 1694

ISSN : 2268-1590

\section{Éditeur :}

Presses universitaires de Paris Sorbonne (PUPS), Société internationale de génétique artistique littéraire et scientifique (SIGALES)

\section{Édition imprimée}

Date de publication : 12 décembre 2016

Pagination : 103-109

ISBN : 9791023105490

ISSN : 1167-5101

\section{Référence électronique}

Johanna Schipper, «Un roman graphique aux rayons X», Genesis [En ligne], 43 | 2016, mis en ligne le 29 octobre 2017, consulté le 30 avril 2019. URL : http://journals.openedition.org/genesis/1694 ; DOI : 10.4000/genesis.1694

Ce document a été généré automatiquement le 30 avril 2019.

Tous droits réservés 


\title{
Un roman graphique aux rayons $\mathrm{X}$
}

\author{
Johanna Schipper
}

Je suis est le contraire de moi.

Valère NOVARINA, L'Espace furieux

1 Nos âmes sauvages est mon second roman graphique autobiographique, publié en 2007 par les éditions Futuropolis. Il relate l'histoire d'un séjour d'immersion chamanique chez les Indiens Shuars (Jivaros) d'Équateur en 2004.

2 Le matériau autobiographique est exigeant car une addition d'anecdotes ne fait pas une histoire. Raconter, c'est articuler une pensée et donner de la cohérence à des éléments hétéroclites. Les réalisateurs le savent bien : le montage est la signature du cinéaste. En bande dessinée, on ne parle pas de montage, mais de découpage. Ce dernier obéit généralement à des codes culturels qui ne constituent pas d'emblée un modus operandi pour l'auteur. Chaque récit porte en lui le secret de sa fabrication.

À partir des anecdotes ramenées du voyage, il m'importait donc de trouver un sens global à l'expérience vécue. Cela a demandé plusieurs années de maturation, durant lesquelles je prenais des notes dans divers carnets, lisais des essais, cherchais des idées. Durant ces mêmes années 2005-2006, je militais activement dans les ONG écologistes et j'étais habituée à aiguiser mon sens de la formule. L'un des dossiers dont j'étais saisie était celui de l'écoblanchiment publicitaire. Je représentais l'association Arutam ${ }^{1}$ aux réunions de l'Alliance pour la planète, aux côtés des Amis de la Terre, de négaWatt, du WWF, de Greenpeace ou du RAP (association Résistance à l'agression publicitaire). À cette époque, les publicitaires s'ingéniaient à trouver des arguments « verts » pour redorer l'image des industries les plus polluantes, comme l'automobile ou le nucléaire. En tant qu'artiste, j'étais particulièrement choquée par ce lifting bio surfant sur la mode écolo. Les slogans donnaient le sentiment que le cynisme avait envahi le paysage urbain.

4 Par exemple, je reportai méticuleusement les arguments orientés des publicités, comme celle pour le Club Med (fig. 1). Je me mis à composer mes propres aphorismes à partir de ces perles, pour en nourrir mon scénario. Cela donna : «L'objet oblige », «Ça crée des liens, des liens sacrés », «L'égotourisme », «Libérez les sabotages!», «Avec vous, je m'étudie », «À mon grand dam, Dame Nature a été condamnée », « Amères zones pour 
Amazonards ", «Les anartistes ", «Plénitude fraîche » ou «pays en voie d'enveloppement » (fig. 2).

Image 2000000900009BC2000003C66A8EEB3B.wmf

Fig. 1

Image 2000000900014B74000001E47B5ED60B.wmf

Fig. 2

Parmi mes lectures figuraient les ouvrages de Jean-Patrick Costa, Jacques Brosse, François Maspero ou Miguel Benasayag. Je décidai alors de ponctuer le déroulement du récit par des citations tirées de leurs livres, car je ne souhaitais pas m'orienter vers une histoire linéaire, ni donner d'indications d'ordre temporel. Seule la coupe de cheveux de l'héroïne devait varier entre le passé et le présent et permettre au lecteur de se repérer dans le temps.

6 À partir de ces notes (que je finis par photocopier et relier ensemble, fig. 3), j'identifiai un certain nombre de scènes clés sur lesquelles je voulais revenir en boucle. Il en émergea une idée de structuration grâce à laquelle il m'était possible de faire ressentir au lecteur l'étrangeté de la transe, où les notions d'espace et de temps semblent abolies. Je décidai de raconter mes visions psychédéliques sous ayahuasca ${ }^{2}$ en faisant des allers-retours entre ce que je vivais durant les cérémonies et mon quotidien de Parisienne. L'ayahuasca fait remonter à la mémoire des éléments du passé qui nous apparaissent sous forme de visions très réalistes. L'usager a l'impression d'ouvrir sa boîte crânienne afin d'en interroger le contenu, voire d'en ré-informer des éléments. En jouant sur l'ambiguïté temporelle et spatiale, j'englobai dans une action unique une période finalement assez longue de ma vie, sans que rien n'indique si le récit se faisait au présent ou était un flashback.

Image 200000090001749F00000118413F5CA2.wmf

Fig. 3 : Notes reliées entre elles

7 J'utilisai pour cela une structure narrative en spirale.

8 Ce premier chemin de fer en colimaçon (fig. 4) fut présenté à l'éditeur Futuropolis en 2005. C'est un document qui, je crois, joua en ma faveur! Dans ce chemin de fer, on distingue 
des petites vignettes qui correspondent chacune aux huit scènes clés qui reviennent en boucle tout au long des soixante-deux planches de l'album.

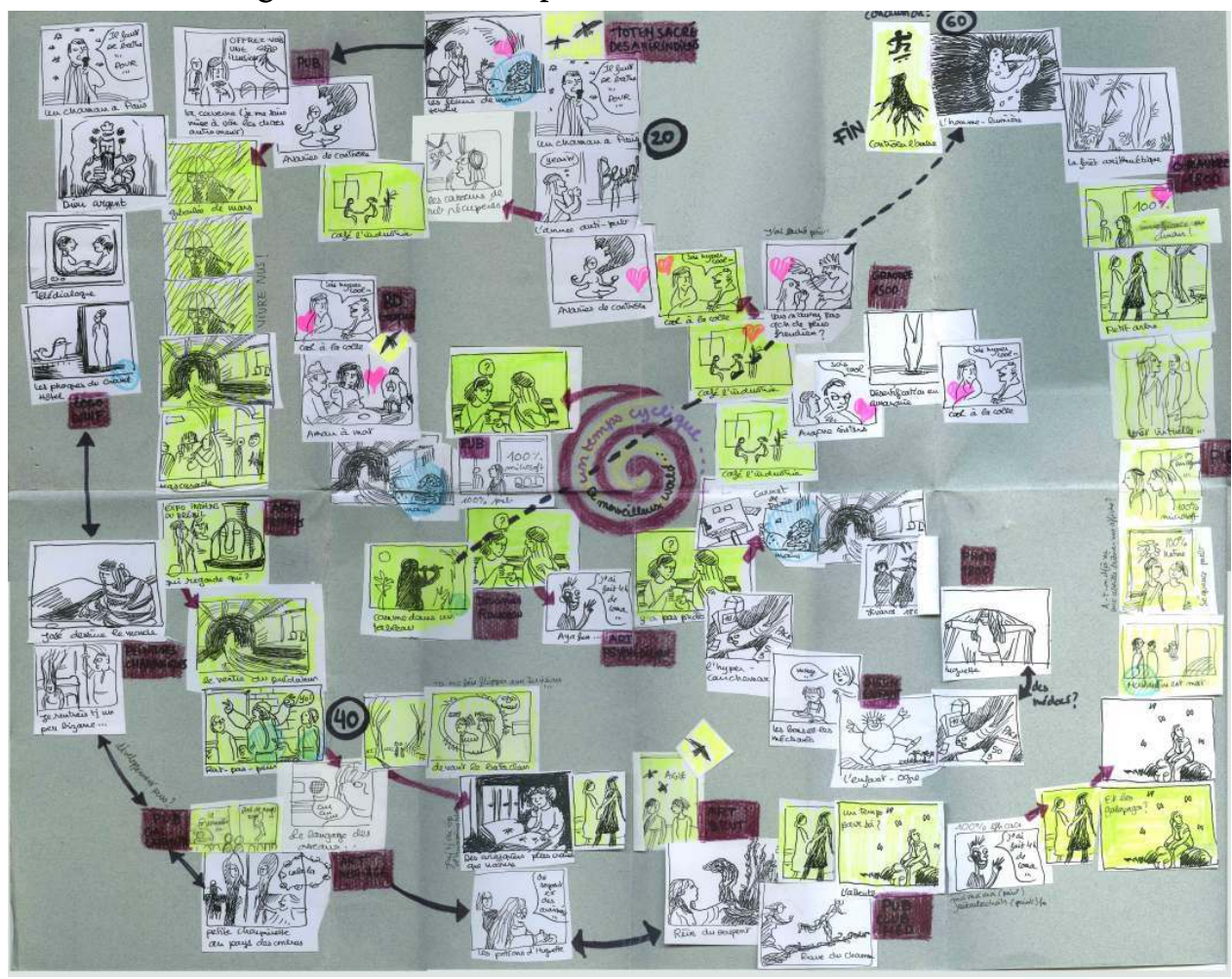

Fig. 4 : Chemin de fer en colimaçon

9 Ces huit scènes clés peuvent être résumées comme suit :

\section{1) L'état visionnaire induit par l'ayahuasca}

10 Il s'agit des visions de la narratrice quand elle participe aux cérémonies chamaniques en Équateur. Ces dernières évoluent et s'enrichissent au fur et à mesure que l'histoire avance. Comme leurs effets sont assez facilement maîtrisables, la narratrice peut, dès qu'elle le souhaite, retrouver son état de veille habituel. C'est un premier dialogue entre deux réalités.

\section{2) Les chamans spécialistes des états modifiés de conscience}

Les chefs spirituels qui font perdurer la tradition shuar ou kichua d'Amazonie ont, par leur pratique, développé une parfaite connaissance de l'état visionnaire provoqué par les psychotropes. Ils sont, par conséquent, habilités à diriger des cérémonies, poser des diagnostiques et manipuler psychiquement ou énergétiquement les gens qui viennent les consulter. Ils portent également parfois aussi une parole militante au-delà de leurs communautés.

\section{3) Les « amazonards »}

12 De nombreux Amérindiens quittent leur mode de vie traditionnel à cause de la déforestation ou par le désir d'accéder à une certaine modernité, et se retrouvent à 
croupir dans les favelas d'Amérique du Sud. Ils sont victimes de la même paupérisation que les punks-à-chiens des villes occidentales, qui rêvent à des alternatives utopistes et se considèrent eux-mêmes comme des Indiens. Mais dans la jungle urbaine, point d'Eden anarchiste. Le métro parisien symbolise parfaitement ces zones grises, ces «amèreszones » que hantent ces personnages : un endroit souterrain où se croisent tous ceux qui sont en transit.

\section{4) L'impossible rencontre avec l'autre}

13 Nos âmes sauvages, c'est également l'histoire d'une rupture sentimentale, dont les soubresauts ponctuent la trajectoire de l'héroïne. La réconciliation impossible avec l'amant, celui qui est au cœur de toutes les projections, met en lumière les tentatives de la narratrice pour compenser le vide d'une solitude vécue comme un mal moderne. Cet amour déçu fait écho au dialogue fragile et complexe entre les cultures autochtones et occidentales.

\section{5) Les fantômes ou les masques de l'émotion}

14 L'héroïne recherche un antidote à son mal-être. Elle avale des couleuvres et emmagasine les frustrations, tout en se construisant le masque de la fille «cool» qui peut tout supporter. Mais, sous l'effet des psychotropes, ce masque prend la forme d'un fantôme dont elle peut enfin se détacher.

\section{6) Le monde des esprits chamaniques}

15 La pensée chamanique est symbolique et s'appréhende dans un espace virtuel (celui des esprits) venant se superposer au monde réel pour l'enrichir et lui donner un sens. Cet espace des esprits peut se concevoir comme un lieu de potentialités dans lequel le passé et l'avenir nous sont accessibles et, par conséquent, modifiables. Dans cet espace, le temps s'appréhende de manière circulaire, toutes les temporalités étant réunies en un seul instant, tous les espaces en un seul lieu.

\section{7) L'amie qui incarne le doute}

Parallèlement aux cérémonies chamaniques, on suit la narratrice dans les rues de Paris, en présence d'une autre femme. Elles parcourent un trajet qui va du Jardin des Plantes au métro Saint-Maur, dans le $\mathrm{XI}^{e}$ arrondissement, tout en discutant de chamanisme, d'écologie et des relations sentimentales de l'héroïne.

\section{8) Le joker}

Le joker est l'acteur du changement de paradigme. C'est le grain de sable qui fait dévier les trajectoires individuelles et collectives de leur prédétermination pour permettre l'émergence de nouveaux possibles.

18 Pour conclure, je choisis de prendre comme fil conducteur la discussion entre la narratrice et son amie. Une dialectique s'installe, nous permettant d'explorer les différents questionnements déjà cités. Parfois, le fil de la discussion s'interrompt, laissant place aux 
points de suspension, aux silences. Mais c'est pour gagner en profondeur à chaque fois que l'on revient sur une scène délaissée quelques pages plus tôt.

Par exemple, page 61 (fig. 5), un zapping rapide s'opère entre les amazonards (3), l'impossible rencontre avec l'autre (4), les fantômes (5) et l'amie qui incarne le doute (7), permettant de montrer à quel point toutes ces scènes sont reliées et revécues en un temps donné par l'héroïne. À ce stade-là du récit, nul besoin d'explication : la juxtaposition en dit plus long qu'une série de cases.

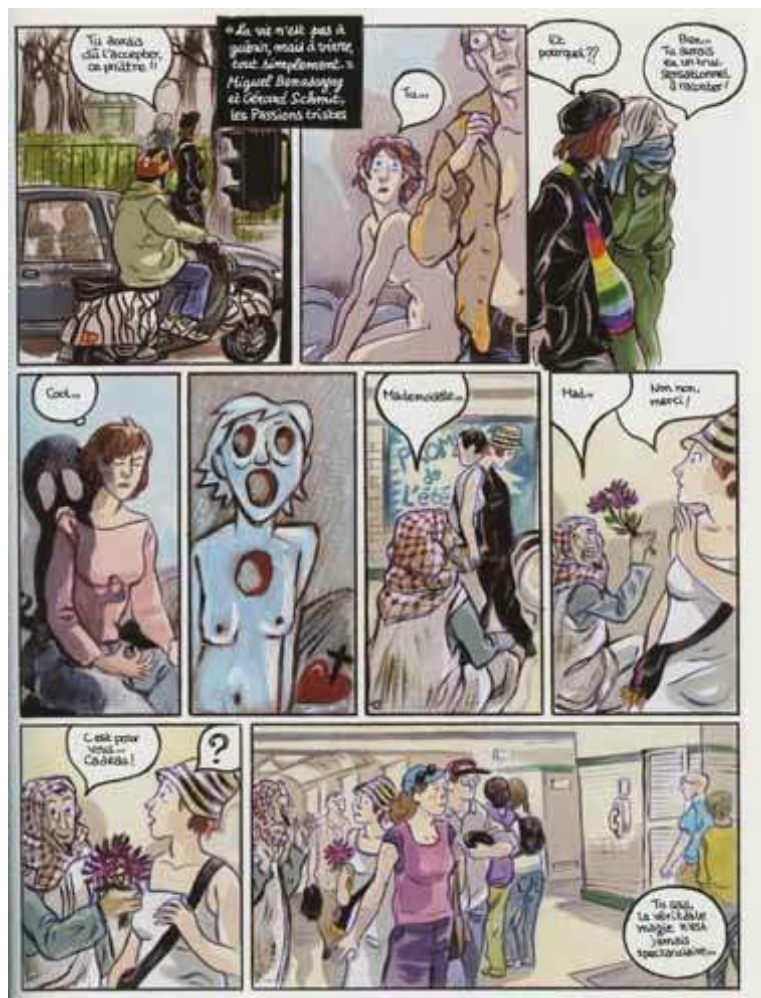

Fig. 5 : Nos âmes sauvages, p. 61 (Futuropolis, 2007)

Et lorsque l'on découvre, en toute fin de l'histoire, que l'amie n'est qu'un personnage imaginaire inventé par la narratrice, la boucle est bouclée et l'on revient au point de départ en Amazonie, en pleine cérémonie chamanique.

\section{NOTES}

1. ONG de défense des peuples autochtones d'Amérique du Sud.

2. Décoction de lianes à effet psychotrope utilisée par les tribus de Haute-Amazonie. 


\section{RÉSUMÉS}

Johanna Schipper présente ici une synthèse des opérations complexes de mise en place du scénario et de la structure en boucle de son second roman graphique, dans lequel interviennent des éléments autobiographiques, politiques et géographiques rebrassés par un jeu vertigineux sur le temps et l'espace.

Johanna Schipper sums up here the elaborate operations of scenario composition and the loop structure of her second graphic novel: it associates autobiographical, political and geographic elements reshuffled in a dizzying play on time and space.

Johanna Schipper presenta aquí una síntesis de las operaciones de puesta en marcha del guión y de la estructura iterativa de su segunda novela gráfica, en la cual intervienen elementos autobiográficos, políticos y geográficos entremezclados gracias a un juego vertiginoso con el tiempo y el espacio.

Johanna Schipper presenta qui una sintesi delle operazioni complesse di preparazione del testo e della struttura circolare del suo secondo romanzo grafico, nel quale intervengono elementi autobiografici, politici e geografici, rimescolati da un gioco vertiginoso sui tempi e lo spazio.

Johanna Schipper präsentiert hier eine Synthese der komplexen Operationen der Schaffung des Szenarios und der Ring-Struktur ihres zweiten graphischen Romans, in dem autobiographische, politische und geographische Elemente durch ein schwindelerregendes Spiel mit Raum und Zeit neu durchgemischt zusammenkommen. 